\title{
ON POINTS OF JACOBIAN RANK $k$
}

\author{
BY \\ P. T. CHURCH
}

1. Introduction. Let $f: M^{n} \rightarrow N^{p}$ be $C^{n}$, where $M^{n}$ and $N^{p}$ are $C^{n}$ manifolds of dimensions $n$ and $p$, respectively. Let $R_{k}(f)$ be the set of points in $M^{n}$ at which the Jacobian matrix of $f$ has rank at most $k$. In $[3,(1.3)]$ it was shown that $\operatorname{dim}\left(f\left(R_{k}\right)\right) \leqq k$. Here the following result is proved:

1.1. ThEOREM. If $i_{*}: \pi_{m}\left(N^{p}-f\left(R_{k}\right)\right) \rightarrow \pi_{m}\left(N^{p}\right)$ is the homomorphism on the mth homotopy groups induced by the inclusion map $i$, then $i_{*}$ is an isomorphism (onto) for $m+k \leqq p-2$, and is onto for $m+k \leqq p-1$.

For example, if $f: E^{2} \rightarrow E^{5}$ is a $C^{2}$ map, then $\pi_{1}\left(E^{5}-f\left(E^{2}\right)\right)=0$. It is easy to see that some differentiability condition is required.

Also the embedding of the set $f(E)$ of [2, Theorem] (proved in [3]) is discussed. For $n \geqq 2, F_{n, d}$ is the canonical open map defined by:

$$
F_{n, d}\left(x_{1}, x_{2}, \cdots, x_{n}\right)=\left(u_{1}, u_{2}, \cdots, u_{n}\right)
$$

where $u_{1}+i u_{2}=\left(x_{1}+i x_{2}\right)^{d}$ and $u_{j}=x_{j}(i=\sqrt{ }-1 ; d=1,2, \cdots ; j=3,4, \cdots, n)$.

1.2. THEOREM. Let $f: M^{n} \rightarrow N^{n}$ be $C^{n}$ and open $(n \geqq 2)$, where $M^{n}$ is compact or $f$ is light. Then there exists a closed set $E_{f}, \operatorname{dim}\left(E_{f}\right) \leqq n-3$, such that for each $x$ in $M^{n}-E_{f}$ there exists a neighborhood of $x$ on which $f$ is topologically equivalent to one of the canonical maps $F_{n, d}(d=1,2, \cdots)$. Moreover, $\pi_{1}\left(N^{n}-f\left(E_{f}\right)\right)$ is isomorphic to $\pi_{1}\left(N^{n}\right)$ under the homomorphism induced by inclusion.

The set $E_{f}$ is (defined to be) the minimal set satisfying the second sentence.

Notation. The set $R_{q}(h)$ may be denoted simply by $R_{q}$, when this causes no ambiguity. The restriction of a function $f$ to a space $X$ will be denoted by $f \mid X$, the interior of $X$ by int $X$, and the closure of $X$ by $X$ or $\mathrm{Cl}[X]$. For $f: X \rightarrow E^{p}$ the coordinate functions will be denoted by $f_{i}(i=1,2, \cdots, p)$.

The distance between two points $x$ and $y$ will be denoted by $d(x, y)$, and the function $d$ may refer (even in the same sentence) to distance on two different metric spaces; from the context, however, the meaning will be clear. An open sphere about $x$ of radius $\varepsilon$ will be denoted by $S(x, \varepsilon)$.

Throughout the paper $M^{n}$ and $N^{p}$ will be manifolds (not necessarily connected) of the indicated dimensions.

Presented to the Society, January 27, 1963; received by the editors January 24, 1963. 
Although the basic ideas of Lemmas 2.1 and 2.4 are not new, the author could not locate the results in the literature, and thus he includes the proofs for completeness.

\section{Preliminary lemmas.}

2.1. Lemma. Let $Q$ be a finite polyhedron, let $\alpha: Q \rightarrow N^{p}$ be continuous, let $v>0$, and let $V$ be an open p-cell in $N^{p}$. Let $P \subset V$ be a finite polyhedron (in a triangulation of $V$ ) with $\operatorname{dim} P+\operatorname{dim} Q \leqq p$, let $E \subset P$ be a compact set with $\operatorname{dim} E+\operatorname{dim} Q \leqq p-1$, and let $W$ be an open neighborhood in $V$ of $P \cap \alpha(Q)$. Then there exists a map $\beta: Q \rightarrow N^{p}$ such that

(1) $d(\alpha, \beta)<v$;

(2) $\beta$ agrees with $\alpha$ on $\alpha^{-1}\left(N^{p}-W\right)$;

(3) $\beta\left(\alpha^{-1}(W)\right) \subset W$; and

(4) $\beta(Q) \cap E=\varnothing$, and $\beta(Q) \cap P$ is a finite set in the simplices of $P$ with largest dimension.

Proof. Let $X$ and $Y$ be open sets with compact closures (in $V$ ) such that $P \cap \alpha(Q) \subset Y, \bar{Y} \subset X$, and $\bar{X} \subset W$. Let $R$ be a finite subpolyhedron of $Q$ such that $\alpha^{-1}(\bar{X}) \subset$ int $R$ and $R \subset \alpha^{-1}(W)$. Choose $\mu>0$ such that $\mu<v$,

(a) $\mu<d(\alpha(R), V-W)$,

(b) $\mu<d(\bar{Y}, V-X)$, and

(c) $\mu<d(\alpha(Q), P \cap(V-Y))$.

Let $\gamma$ be a simplical approximation to $\alpha \mid R$ such that $d(\alpha \mid R, \gamma)<(1 / 3) \mu$. By a general position argument we may move the polyhedron $\gamma(R)$ slightly, defining a simplicial map $\delta: R \rightarrow V$ such that $d(\alpha \mid R, \delta)<(2 / 3) \mu$ and either (i) $\delta(R) \cap P=\varnothing$, in case $\operatorname{dim} P+\operatorname{dim} Q<p$, or (ii) $\delta(R) \cap P$ is a finite set of points disjoint from the $((\operatorname{dim} P)-1)$-skeleton of $P$, in case $\operatorname{dim} P+\operatorname{dim} Q=p$. In case (i), let the map $\lambda: R \rightarrow V$ be $\delta$. In case (ii) $E$ is nowhere dense in $P[5, \mathrm{p} .46]$; thus by moving $\delta(R)$ slightly we may define $\lambda$ such that $d(\alpha \mid R, \lambda)<\mu$ and $\lambda(R) \cap E=\varnothing$.

Define $\beta$ by: $\beta=\alpha$ on $Q-$ int $R, \beta=\lambda$ on $\alpha^{-1}(\bar{X})$, and $\beta=t \cdot \lambda+(1-t) \alpha$ elsewhere, where

$$
t(x)=\frac{d(x, Q-\operatorname{int} R)}{d(x, Q-\operatorname{int} R)+d\left(x, \alpha^{-1}(X)\right)} .
$$

That $\beta$ is continuous and satisfies conclusions (1) and (2) is immediate. For (3), it suffices to prove that $\beta(R) \subset W$, which follows from (a) and the fact that $d(\alpha, \beta)<\mu$. For (4) $\beta\left(R-\alpha^{-1}(X)\right) \subset V-Y$ by (b), and $\beta\left(R-\alpha^{-1}(X)\right) \cap P=\varnothing$ by (c). Thus $\beta\left(Q-\alpha^{-1}(X)\right) \cap P=\varnothing$, and (4) follows.

2.2. Remark. Let $f: M^{n} \rightarrow E^{p}$ be a $C^{n}$ map, where $M^{n}$ is a $C^{n}$ manifold. There exists a countable number of open Euclidean coordinate neighborhoods $N_{i}$ which cover $M^{n}$, and $C^{n}$ diffeomorphisms $h^{i}: N_{i} \rightarrow E^{n}(i=1,2, \cdots)$. We may suppose that the sets $h^{i}\left(N_{i}\right)$ are mutually disjoint; let $V=\bigcup_{=1}^{\infty} h^{i}\left(N_{i}\right)$, and define $g: V \rightarrow E^{1}$ by the restriction $g \mid h^{i}\left(N_{i}\right)=f\left(\left(h^{i}\right)^{-1}\right)$. Then $g\left(R_{0}(g)\right)=f\left(R_{0}(f)\right)$. 
If $X_{j}$ is the set of critical points of $g_{j}$ (the points at which all first partials are zero), then tbhe Lebesgue measure of $g_{j}\left(X_{j}\right)$ is zero $[8$, p. $68,(4.3)](j=1,2, \cdots, p)$. Since $R_{0}(g)=\bigcap_{j=1}^{p} X_{j}$, for each $\varepsilon>0$ there exist real numbers $a_{j}^{i}(i=0, \pm 1, \cdots$; $j=1,2, \cdots, p)$ such that $\left|a_{j}^{i}-a_{j}^{i+1}\right|<\varepsilon$ and the $(p-1)$-planes $g_{j}=a_{j}^{i}$ are disjoint from $g\left(R_{0}(g)\right)\left(=f\left(R_{0}(f)\right)\right)$.

2.3. REMARK. In $[3,(1.1)]$, if the dependent variables are ordered so that $\operatorname{det}\left[D_{j} h_{i}\right](\bar{x}) \neq 0(i=1,2, \cdots, q$; some $q$ values of $j)$, then $k^{2}$ may be chosen to be the identity; thus $g^{-1}(\alpha)$ is actually $h^{-1}(\alpha)$. To see this, replace the version of the rank theorem used in [3] by [7, pp. 7-8].

2.4. Lemma. If $W_{i}$ is an open cover of the locally compact metric space $R$, then there exists a cover $X_{i}$ closed in $R$ such that $X_{i} \subset W_{i}(i=1,2, \cdots, L)$.

Proof. If $L=1$, then $X_{1}=W_{1}$. Otherwise we proceed by induction. Let $Y=R-\bigcup_{i \neq 1} W_{i}$. For each $y \in Y$ let $S\left(y, \varepsilon^{y}\right)$ be a sphere in $R$ with compact closure, $d\left(y, R-W_{1}\right)>2 \varepsilon^{y}$. Let $S$ be the union of the closed spheres, and let $X_{1}=S$ (closure in $R$ ). Suppose that $x^{j} \rightarrow x$, where $x^{j} \in S$ and $x \in R$, and that $x^{j} \in \mathrm{Cl}\left[S\left(y^{j}, \varepsilon^{j}\right)\right]$. If $\varepsilon^{j} \rightarrow 0$, then $y^{j} \rightarrow x$. Thus $x$ is in thle cosed set $Y$, and thus is in $W_{1}$. If $\varepsilon^{j} \nrightarrow 0$, then there is a subsequence (which we still call $\varepsilon^{j}$ ) such that $\varepsilon^{j} \geqq \varepsilon>0$. For some $J d\left(x^{J}, x\right)<\varepsilon$, and thus $d\left(y^{J}, x\right)<2 \varepsilon^{J}$. Hence $x \notin R-W_{1}$. As a result, $Y \subset \operatorname{int}\left(X_{1}\right)$ and $X_{1} \subset W_{1}$.

Since $R$-int $\left(X_{1}\right)$ is locally compact with open cover $W_{i} \cap\left(R-\operatorname{int}\left(X_{1}\right)\right)$ $(i=2,3, \cdots, L)$, there exists by inductive hypothesis a closed (in $R-\operatorname{int}\left(X_{1}\right)$ ) cover $X_{i}$. The sets $X_{i}(i=1,2, \cdots, L)$ are the desired closed covers of $R$.

Definition. An open (resp., closed) coordinate p-rectangle in $E^{p}$ is the set of points $\left(x_{1}, x_{2}, \cdots, x_{p}\right)$ such that $a_{i}<x_{i}<b_{i}$ (resp., $a_{i} \leqq x_{i} \leqq b_{i}$ ) for some real $a_{i}$ and $b_{i}(i=1,2, \cdots, p)$.

2.5. LemMA. Let $L$ be a compact subset of $E^{p}$, and let $\left\{T_{i}\right\}(i=1,2, \cdots, r)$ be a cover of $L$ by open coordinate p-rectangles. Then there exist a bounded open (in $\left.E^{p}\right)$ cover $\left\{H_{j}\right\}(j=1,2, \cdots, s)$ of $L$ and sets $\mathscr{N}_{j} \subset\{1,2, \subset, r\}$ such that:

(1) if $i \in \mathscr{N}_{j}$, then $H_{j} \subset T_{i}$;

(2) if $H_{j} \cap H_{k} \neq \varnothing$ and $j>k$, then $\mathscr{N}_{k} \subset \mathscr{N}_{j}$;

(3) there exists $\delta>0$ such that each sphere $S(y, \delta)$ meets at most $2^{p}$ sets of the cover $H_{j}$;

(4) there is a cover $\left\{G_{j}\right\}$ of $L$ by closed coordinate p-rectangles with $G_{j} \subset H_{j}$ $(j=1,2, \cdots, s)$.

Proof. There is a cover $\left\{U_{i}\right\}$ of $L$ by closed coordinate $p$-rectangles with $U_{i} \subset T_{i}$; let $\mathscr{U}(\{i\})=\left\{U_{i}\right\}(i=1,2, \cdots, r)$. Since $U_{h} \cap U_{i}$ is compact, it has a finite cover by open coordinate $p$-rectangles each of whose closures is contained in $T_{h} \cap T_{i}$ (if $U_{h} \cap U_{i}=\varnothing$, then the cover is empty). Let $\mathscr{U}(\{h, i\})$ be the set of these closed $p$-rectangles, and let $U(\{h, i\})$ be their union $(h \neq i ; h, i=1,2, \cdots, r)$. 
We continue by induction. Suppose that, for all $\mathscr{I} \subset\{1,2, \cdots, r\}$ with at most $A$ elements, $\mathscr{U}(\mathscr{I})$ and $U(\mathscr{I})$ have been defined such that :

$(\mathrm{A}, 1) U(\mathscr{I}) \subset \bigcap T_{i}(i \in \mathscr{I})$.

$(\mathrm{A}, 2)$ Suppose that $x \in U(\mathscr{J}) \cap U(\mathscr{K})$, where each of $\mathscr{J}$ and $\mathscr{K}$ has less than $A$ elements, $\mathscr{J} \notin \mathscr{K}$ and $\mathscr{K} \notin \mathscr{J}$. Then $x$ is in the interior of a $p$-rectangle of $\mathscr{U}(\mathscr{I})$, for some $\mathscr{I}$ with more elements than either $\mathscr{J}$ or $\mathscr{K}$. (In general, this $\mathscr{I}$ is not unique.)

Let $\mathscr{I} \subset\{1,2, \cdots, r\}$ with $A+1$ elements be given. For each pair of subsets $\mathscr{J}$ and $\mathscr{K}$ of $\mathscr{I}$ such that $\mathscr{J} \notin \mathscr{K}, \mathscr{K} \notin \mathscr{J}$, and $\mathscr{J} \cup \mathscr{K}=\mathscr{I}$, the compact set $U(\mathscr{J}) \cap U(\mathscr{K})$ has a finite cover (possibly empty) by open coordinate $p$-rectangles, each of whose closures is contained in $\bigcap T_{i}(i \in \mathscr{I})$, by $(\mathrm{A}, 1)$. Let $\mathscr{U}(\mathscr{I})$ be the set of all these closed $p$-rectangles for all such pairs $\mathscr{J}$ and $\mathscr{K}$, and let $U(\mathscr{I})$ be the union of the $p$-rectangles of $\mathscr{U}(\mathscr{I})$. Then the sets $\mathscr{U}(\mathscr{I})$ and $U(\mathscr{I})$ for $\mathscr{I}$ with $A+1$ elements satisfy $(A+1,1)$ and $(A+1,2)$. Thus $\mathscr{U}(\mathscr{I})$ and $U(\mathscr{I})$ are defined for all $\mathscr{I} \subset\{1,2, \cdots, r\}$.

Consider those $(p-1)$-planes each of which contains a $(p-1)$-face of a $p$ rectangle of a set $\mathscr{U}(\mathscr{I})(\mathscr{I} \subset\{1,2, \cdots, r\})$. The planes bound a finite number of closed coordinate $p$-rectangles; let $G_{j}(j=1,2, \cdots, s)$ be those each of which is contained in a set $U(\mathscr{O})$. Then $\left\{G_{j}\right\}$ is a cover of $L$ of order at most $2^{p}$.

Let $\mathscr{N}_{j}$ be a maximal subset $\mathscr{I}$ of $\{1,2, \cdots, r\}$ such that $G_{j} \subset U(\mathscr{I})$; it follows from $(r, 2)$ that there is only one maximal set. We may suppose that the set $\{1,2, \cdots, s\}$ of indices of $G_{j}$ has been ordered so that $k<j$ implies that $\mathscr{N}_{k}$ does not have more elements than $\mathscr{N}_{j}$. That the $G_{j}$ satisfy conclusion (1) follows from $(r, 1)$. Suppose that there exists $x \in G_{j} \cap G_{k}$, where $j>k$ and $\mathscr{N}_{k} \notin \mathscr{N}_{j}$; then $x \in U\left(\mathscr{N}_{j}\right) \cup U\left(\mathscr{N}_{k}\right)$, contradicting $(r, 2)$ and the definition (maximality) of $\mathscr{N}_{j}$. Thus the sets $G_{j}$ satisfy conclusion (2). Choose $\delta>0$ such that each edge of each $p$-rectangle $G_{j}$ has length greater that $4 \delta$, and let

$$
H_{j}=\left\{x \in E^{p}: d\left(x, G_{j}\right)<\delta \text { and } x \in T_{i}\left(i \in \mathscr{N}_{i}\right)\right\}
$$

That the sets $H_{j}(j=1,2, \cdots, s)$ satisfy the conclusions is immediate.

\section{The proof of (1.1).}

3.1. Lemma. Let $h: U \rightarrow E^{p}$, where $U$ is open in $E^{n}, h$ is $C^{n}$, and $E^{p}$ is a Euclidean coordinate neighborhood of $N^{p}$. Suppose that some $q \times q$ subdeterminant $D$ of the Jacobian determinant of $h$ is nonzero on $U(q=1,2, \cdots ;$ no assumption is made if $q=0$ ), and that $Y$ is a compact subset of $U$. Let $Q$ be a finite polyhedron such that $q+\operatorname{dim} Q \leqq p-1$, let $\alpha: Q \rightarrow N^{p}$ be continuous, and let $\Delta \subset Q$ be compact with

$$
\alpha(\Delta) \cap h\left(Y \cap R_{q}(h)\right)=\varnothing .
$$

Then, given $\eta>0$, there exists a map $\gamma: Q \rightarrow N^{p}$ such that 
(1) $d(\alpha, \gamma)<\eta$,

(2) $\gamma$ agrees with $\alpha$ on $\Delta \cup \alpha^{-1}\left(N^{p}-E^{p}\right)$, and

(3) $\gamma(Q) \cap h\left(Y \cap R_{q}(h)\right)=\varnothing$.

Proof. By (2.2) there exist in $E^{p}$ a finite number of disjoint closed coordinate $p$-rectangles of diameter less than $\eta / 2$, such that the union $K$ is disjoint from $\alpha(\Delta)$ and $h\left(R_{0}(h) \cap Y\right) \subset \operatorname{int} K$. Let $\beta$ be the map given by (2.1) for $\alpha, V=E^{p}$, $E=P$ the union of the center points of the $p$-rectangles of $K, W=$ int $K$, and $v=\eta / 2$. Let $g: N^{L}-P \rightarrow N^{p}-$ int $K$ be the identity on $N^{p}-$ int $K$, and, on each rectangle of $K$, projection from the center point to the boundary. Then $\gamma=g \beta$ is the desired map for $q=0$.

For $q=1,2, \cdots$ we may as well suppose that the dependent variables of $D$ are indexed by $1,2, \cdots, q$, and write $E^{p}$ as $E^{q} \times E^{p-q}$. Let $\pi: E^{p} \rightarrow E^{q}$ be the projection map. By [3, (1.1)] and (2.3), for each $w \in E^{q}, h^{-1}\left(\{w\} \times E^{p-q}\right)$ is a $C^{n}(n-q)$ manifold $A_{w}$ (not necessarily connected). Also $R_{q}(h) \cap A_{w}=R_{0}\left(h \mid A_{w}\right)$, where $h \mid A_{w}$ is the restriction map; hereafter, $R_{q}$ denotes $R_{q}(h)$.

By (2.2) and the above remark, for each $w \in E^{q}$, there exists in $E^{p-q}$ a finite union $K(w)$ of mutually disjoint closed coordinate $(p-q)$-rectangles of diameter less than $\eta \cdot 2^{-q-1}$, such that

We may suppose that

$$
\begin{aligned}
h\left(R_{q} \cap A_{w} \cap Y\right) & =h\left(R_{q} \cap Y\right) \cap\left(\{w\} \times E^{p-q}\right) \\
& \subset(\{w\} \times \operatorname{int}(K(w)) .
\end{aligned}
$$

$$
\eta \cdot 2^{-q-1}<d\left(h\left(R_{q} \cap Y\right), \alpha(\Delta)\right) .
$$

Since $h\left(R_{q} \cap Y\right)$ is compact, for each $w \in E^{q}$ and for $v$ sufficiently near $w$,

$$
h\left(R_{q} \cap Y\right) \cap\left(\{v\} \times E^{p-q}\right) \subset(\{v\} \times \operatorname{int}(K(w))) .
$$

Thus there exists an open coordinate $q$-rectangle neighborhood $T(w)$ such that (*) holds for each $v \in \bar{T}(w)$, and $\alpha(\Delta) \cap(\bar{T}(w) \times K(w))=\varnothing$. The compact subset $\pi\left(h\left(R_{q} \cap Y\right)\right)$ in $E^{q}$ has a finite subcover $\left\{T\left(w^{j}\right)\right\}(j=1,2, \cdots, r)$. Let $G_{i}, H_{i}, \mathscr{N}_{i}$ $(i=1,2, \cdots, s)$ and $\delta>0$ be the sets and number given by (2.5) for $L=\pi\left(h\left(R_{q} \cap Y\right)\right)$; we may suppose that $\delta<\eta / 2$. Let $K_{i}$ be the intersection of $K\left(w^{j}\right)$ for $j \in \mathscr{N}_{i}$. Then $\{w\} \times K_{i}$ is also the finite union (possibly empty) of mutually disjoint closed coordinate $(p-q)$-rectangles, containing in its interior

$$
h\left(R_{q} \cap Y\right) \cap\left(\{w\} \times E^{p-q}\right)
$$

for each $w$ in $H_{i}$ (by relation (*) above, and by conclusion (1) of (2.5)). Thus

$$
h\left(Y \cap R_{q}\right) \subset \bigcup_{i=1}^{s}\left(G_{i} \times \operatorname{int}\left(K_{i}\right)\right)
$$

Also $\alpha(\Delta) \cap\left(\bar{H}_{i} \times K_{i}\right)=\varnothing(i=1,2, \cdots, s)$. 
Let $S_{i}$ be the union of the center points of the $(p-q)$-rectangles in $K_{i}$. Then $P_{i}=G_{i} \times S_{i}$ is a $q$-dimensional finite subpolyhedron in (the triangulated set) $E^{p}(i=1,2, \cdots, s)$. Let $\beta^{1}$ be the map given by (2.1) for $\alpha, \nu=\delta \cdot 2^{-q}, E=P=P_{1}$ and $W=H_{1} \times \operatorname{int}\left(K_{1}\right)$. Thus $\beta^{1}(Q) \cap P_{1}=\varnothing$ by conclusion (4) of (2.1).

Given any map $\beta^{i}: Q \rightarrow N^{p}$ such that $\beta^{i}(Q) \cap P_{i}=\varnothing$, we now define a map $\gamma^{i}: Q \rightarrow N^{p}(\mathrm{i}=1,2, \cdots, s)$. Choose $\mu_{i}>0, \mu_{i}$ less than the minimum of $d\left(\beta^{i}(Q), P_{i}\right)$, and $d\left(G_{i}, E^{q}-H_{i}\right)$; and let

$$
F_{i}=\left\{w \in E^{q}: d\left(w, G_{i}\right)<\mu_{i}\right\} .
$$

Thus $P_{i} \subset F_{i} \times S_{i}$, and $\beta^{i}(Q) \cap\left(F_{i} \times S_{i}\right)=\varnothing$. We will define a map $g^{i}$ such that its domain and range are $N^{p}-\left(F_{i} \times S_{i}\right)$, and on $N^{p}-\left(F_{i} \times K_{i}\right) g_{i}$ is the identity map. For each $w \in F_{i}$,

$$
g^{i}\left(\{w\} \times\left(E^{p-q}-S_{i}\right)\right) \subset\left(\{w\} \times\left(E^{p-q}-S_{i}\right)\right),
$$

i.e., $\pi g^{i}=\pi$. For each $w \in G_{i}, g^{i}$ is the natural retraction map, i.e., for $x \in E^{p-q}-K_{i}, g^{i}$ is the identity map, and for $x$ in a $(p-q)$-rectangle of $K_{i}$, $g^{i}$ projects $x$ from the center of the rectangle to the boundary. For $w \in F_{i}-G_{i}$ replace each (coordinate) $(p-q)$-rectangle of $K_{i}$, say of side $t$, with a coordinate $(p-q)$-rectangle of the same center and side $\left(\mu_{1}-d\left(w, G_{i}\right)\right)\left(\mu_{1}\right)^{-1} t$. Define $g^{i}$ on $\{w\} \times\left(E^{p-q}-S_{i}\right)$ analogously to its definition for $w \in G_{i}$. Then $g^{i}$ is continuous.

We shall show that the map $\gamma^{i}$ defined by $\gamma^{i}=g^{i} \beta^{i}(i=1,2, \cdots, s)$ satisfies the following properties:

(a) $\pi \gamma^{i}=\pi \beta^{i}$;

(b) $d\left(\gamma^{i}, \beta^{i}\right)<\eta \cdot 2^{-q-1}$;

(c) $\gamma^{i}=\beta^{i}$ on $Q-\left(\beta^{i}\right)^{-1}\left(H_{i} \times K_{i}\right)$ and $\gamma^{i}\left(\left(\beta^{i}\right)^{-1}\left(H_{i} \times K_{i}\right)\right) \subset H_{i} \times K_{i}$;

(d) if $\beta^{i}(Q) \cap\left(G_{j} \times \operatorname{int}\left(K_{j}\right)\right)=\varnothing(j=1,2, \cdots, i-1)$, then

$$
\gamma^{i}(Q) \cap\left(G_{m} \times \operatorname{int}\left(K_{m}\right)\right)=\varnothing \quad(m=1,2, \cdots, i) .
$$

Property (a) holds because $\pi g^{i}=\pi$, (b) because the diameters of the $(p-q)$ rectangles of $K_{i}$ are less than $\eta \cdot 2^{-q-1}$, and (c) is immediate.

Property (d) is clear for $m=i$. For those $j$ such that $H_{i} \cap H_{j}=\varnothing$, (d) follows from (c). If $H_{i} \cap H_{j} \neq \varnothing(j=1,2, \cdots, i-1)$, then $\mathscr{N}(j) \subset \mathscr{N}(i)$ (by conclusion (2) of (2.5)), so that $K_{i} \subset K_{j}$. Suppose that $\gamma^{i}(Q) \cap\left(G_{j} \times \operatorname{int}\left(K_{j}\right)\right) \neq \varnothing$. Then, by (c) and the hypothesis of (d),

$$
\gamma^{i}\left(\left(\beta^{i}\right)^{-1}\left(H_{i} \times \operatorname{int}\left(K_{i}\right)\right)\right) \cap\left(G_{j} \times \operatorname{int}\left(K_{j}\right)\right) \neq \varnothing .
$$

Since $\pi g^{i}=\pi$ and $g^{i}=\gamma^{i}\left(\beta^{i}\right)^{-1}$,

$$
\gamma^{i}\left(\left(\beta^{i}\right)^{-1}\left(\left(H_{i} \cap G_{j}\right) \times \operatorname{int}\left(K_{i}\right)\right)\right) \cap\left(G_{j} \times \operatorname{int}\left(K_{j}\right)\right) \neq \varnothing .
$$

Now $K_{i} \subset K_{j}$, so that 


$$
\gamma^{i}\left(\left(\beta^{i}\right)^{-1}\left(G_{j} \times \operatorname{int}\left(K_{j}\right)\right)\right) \cap\left(G_{j} \times \operatorname{int}\left(K_{j}\right)\right) \neq \varnothing ;
$$

by the hypothesis of (d), we have a contradiction. Thus (d) is satisfied.

A map $\gamma^{1}$ has been defined. We continue by induction. A set of maps $\left\{\gamma_{i}\right\}$ ( $i=1,2, \cdots, I$ ) is said to satisfy property $\mathscr{P}_{I}$ if

(a) $d\left(\pi \gamma^{i}, \pi \gamma^{i-1}\right)<\delta \cdot 2^{-q}\left(\gamma^{0}=\alpha\right)$,

(b) $d\left(\gamma^{i}, \gamma^{i-1}\right)<\eta \cdot 2^{-q}$,

(c) $\gamma^{i}=\gamma^{i-1}$ on $\alpha^{-1}\left(N^{p}-E^{p}\right) \cup \Delta \cup\left(Q-\left(\gamma^{i-1}\right)^{-1}\left(H_{i} \times K_{i}\right)\right)$,

(d) $\gamma^{i}(Q) \cap\left(G_{j} \times \operatorname{int}\left(K_{j}\right)\right)=\varnothing \quad(j=1,2, \cdots, i ; i=1,2, \cdots, I)$. The set $\left\{\gamma^{1}\right\}$ satisfies $\mathscr{P}_{I}$ (for (c) remember that $\left.\alpha(\Delta) \cap\left(\bar{H}_{i} \times K_{i}\right)=\varnothing\right)$. Given $\left\{\gamma^{i}\right\}$ $(i=1,2, \cdots, I)$ satisfying $\mathscr{P}_{I}$, we will now define $\gamma^{I+1}$ such that $\left\{\gamma^{i}\right\}$ $(i=1,2, \cdots, I+1)$ satisfies $\mathscr{P}_{I+1}$.

Let $X=P_{I+1} \cap \gamma^{I}(Q)$. Since $P_{I+1}=G_{I+1} \times S_{I+1}$, if $H_{i} \cap H_{I+1}=\varnothing$, then $X \cap\left(G_{i} \times K_{i}\right)=\varnothing \quad(i=1,2, \cdots, I)$. If $H_{i} \cap H_{I+1} \neq \varnothing$, then $\mathscr{N}_{i} \subset \mathscr{N}_{I+1}$ by conclusion (2) of (2.5), so that $K_{I+1} \subset K_{i}$. Since $P_{I+1} \subset\left(E^{q} \times \operatorname{int}\left(K_{I+1}\right)\right)$, $X \cap\left(G_{i} \times \operatorname{bdy}\left(K_{i}\right)\right)=\varnothing$. By $\mathscr{P}_{I},(\mathrm{~d}), X$ is disjoint from $G_{i} \times \operatorname{int}\left(K_{i}\right)$ and thus from $G_{i} \times K_{i}$. Thus $X \subset W$, where

$$
W=\left(H_{I+1} \times \operatorname{int}\left(K_{I+1}\right)\right)-\bigcup_{i=1}^{I}\left(G_{i} \times K_{i}\right) .
$$

Apply (2.1) for $\alpha=\gamma^{I}, E=P=P_{I+1}, W$, and $v<\delta \cdot 2^{-q}$, calling the resulting $\operatorname{map} \beta^{I+1}$. Then $\beta^{I+1}(Q) \cap P_{I+1}=\varnothing$. Let $\gamma^{I+1}$ be the map given for $\beta^{I+1}$.

That $\left\{\gamma^{i}\right\}(i=1,2, \cdots, I+1)$ satisfies $\mathscr{P}_{I+1}$ follows from the corresponding properties for $\left\{\gamma^{i}\right\}(i=1,2, \cdots, I)$, the definitions of $\beta^{I+1}$ and of $\gamma^{I+1}$ from $\beta^{I+1}$, and the fact that $\delta<\eta / 2$. For (d), observe that $\beta^{I+1}=\gamma^{I}$ on $\left(\gamma^{I}\right)^{-1}\left(N^{p}-W\right)$ and $\beta^{I+1}(W) \subset W$. Thus $\beta^{I+1}(Q) \cap\left(G_{i} \times\right.$ int $\left.\left(K_{i}\right)\right)=\varnothing$ $(i=1,2, \cdots, I)$ by $\mathscr{P}_{I},(\mathrm{~d})$.

The desired map $\gamma$ is $\gamma^{s}$. Given any point $\alpha(x)$ in $E^{p}, S(\pi \alpha(x), \delta)$ (in $E^{q}$ ) meets $H_{i}$ for at most $2^{q}$ different $i$, by the definition of $\delta$. As a result, by $\mathscr{P}_{s}$, (a) and (c), $\pi \gamma^{i}(x) \in S(\pi \alpha(x), \delta) \quad(i=1,2, \cdots, s)$. By $\mathscr{P}_{s}$, (b) and the definition of $\delta$, $d(\gamma, \alpha)<\left(\eta \cdot 2^{-q}\right) \cdot 2^{q}=\eta$. The remaining properties of $\gamma$ are immediate from $P_{s}$, and the fact that

$$
h\left(Y \cap R_{q}\right) \subset \bigcup_{i=1}^{s}\left(G_{i} \times \operatorname{int}\left(K_{i}\right)\right)
$$

3.2. LEMMA. Let $f: M^{n} \rightarrow N^{p}$ be a $C^{n}$ map, and let $k$ be a non-negative integer and $Q$ a finite polyhedron such that $k+\operatorname{dim} Q \leqq p-1$. Let $\varepsilon$ be positive, let $\Delta \subset Q$ be compact, and let $\alpha: Q \rightarrow N^{p}$ be a map such that $\alpha(\Delta) \cap f\left(R_{k}(f)\right)=\varnothing$. Then there exists a map $\gamma: Q \rightarrow N^{p}$ such that $\gamma(Q) \cap f\left(R_{k}(f)\right)=\varnothing, \gamma|\Delta=\alpha| \Delta$, and $d(\alpha, \gamma)<\varepsilon$.

Proof. Choose compact sets $A_{i} \subset M^{n}$, and Euclidean coordinate neighbor- 
hoods $U_{i} \subset M^{n}$ and $V_{i} \subset N^{p}(i=1,2, \cdots)$ such that $R_{k} \subset \bigcup_{i} A_{i}, A_{i} \subset U_{i}$, and $f\left(U_{i}\right) \subset V_{i}$. For each $i$ and $q(q=1,2, \cdots, \min (n, k))$ let $D_{i, q, j}$ be an enumeration of the $q \times q$ subdeterminants of the Jacobian determinant of $f \mid U_{i}$; let $W_{i, q, j}$ be the set of points in $U_{i}$ at which $D_{i, j, q} \neq 0$. Then each set $R_{q} \cap W_{i, q, j}$ is open in $R_{q}$, and $R_{q} \cap \bigcup_{j} W_{i, q, j}=U_{i} \cap\left(R_{q}-R_{q-1}\right)$. Let $X_{i, q, j}$ be the sets closed in $A_{i} \cap\left(R_{q}-R_{q-1}\right)$, given by (2.4), such that

$$
X_{i, q, j} \subset A_{i} \cap\left(R_{q}-R_{q-1}\right) \cap W_{i, q, j}
$$

and $\bigcup_{j} X_{i, q, j}=A_{i} \cap\left(R_{q}-R_{q-1}\right)$; let $X_{i, 01}=A_{i} \cap R_{0}$.

We may order the sets $X_{i, q, j}$ lexicographically (for each $i$, there are only a finite number of $q$ and $j)$, and rename them $C_{s}(s=1,2, \cdots)$. Some of the sets may be empty. For each $S$ there exist $L, Q$, and $T(S, T=1,2, \cdots ; T \leqq S ; L, Q=0,1, \cdots)$ such that

$$
\bigcup_{s=1}^{T} C_{s}=\left[\left(\bigcup_{i=1}^{L} A_{i}\right) \cap R_{k}\right] \cup\left(A_{L+1} \cap R_{Q}\right)
$$

and $\bigcup C_{s}(s=T+1, T+2, \cdots, S)$ is the union of sets $X_{L+1, Q+1, j}$ for none, some or all of the possible $j$. Since each of the limit points (in $M^{n}$ ) of the second set is either in that set or in $A_{L+1} \cap R_{Q}, \bigcup C_{s}(s=1,2, \cdots, S)$ is compact. Let $G_{S}$ denote this set.

Let $\gamma^{1}$ be the map given by (3.1) for $h=f \mid U_{1}, E^{p}=V_{1}, q=0, Y=A_{1}$, and $\eta=\varepsilon / 2$. Let $\varepsilon_{1}=\varepsilon / 2$, and let $\delta_{1} \leqq d\left(\gamma^{1}(Q), f\left(C_{1}\right)\right), \delta_{1}>0$.

Suppose that a set of maps $\gamma^{s}: Q \rightarrow N^{p}$ and numbers $\varepsilon_{s}>0$ and $\delta_{s}>0$ $(s=1,2, \cdots, S)$ have been defined such that

$(\mathrm{S}, 1) \gamma^{s}\left|\Delta=\gamma^{s-1}\right| \Delta\left(\gamma^{0}=\alpha\right)$,

$(\mathrm{S}, 2) d\left(\gamma^{s}(Q), f\left(G_{s}\right)\right) \geqq \delta_{s}$, and

$(\mathrm{S}, 3) d\left(\gamma^{s}, \gamma^{s-1}\right)<\varepsilon_{s}$, where $\varepsilon_{s}$ is the minimum of $\varepsilon \cdot 2^{-s}$ and $\delta_{r} \cdot 2^{-s+r-1}$ $(r=1,2, \cdots, s-1)$.

Let $\varepsilon_{S+1}$ be defined as in $(S, 3)$, let

$$
Z=\left\{x \in N^{p}: d\left(x, f\left(G_{s}\right)\right)<\delta_{s} / 2\right\},
$$

and let $Y=\left(N^{p}-Z\right) \cap f\left(C_{S+1}\right)$. Since $\bar{C}_{S+1}-C_{S+1} \subset G_{S}, Y$ is compact. Let $\gamma^{s+1}$ be the map given by (3.1) for $h=f \mid W_{i, q, j}$ (where $\left.C_{S+1}=X_{i, q, j}\right), E^{p}=V_{i}$, the above set $Y, \alpha=\gamma^{s}$, and $\eta=\varepsilon_{S+1}$. Then $\gamma^{s+1}\left|\Delta=\gamma^{s}\right| \Delta$, and $\gamma^{s+1}(Q) \cap f(Y)=\varnothing$. Since $d\left(\gamma^{S+1}, \gamma^{s}\right)<\varepsilon_{S+1} \leqq \delta_{S} / 4, \gamma^{S+1}(Q) \cap Z=\varnothing$. Thus there exists $\delta_{S+1}>0$ such that

$$
d\left(\gamma^{S+1}(Q), f\left(G_{S+1}\right)\right) \geqq \delta_{S+1} .
$$

Let $\gamma$ be the limit of the maps $\gamma^{s}(s=1,2, \cdots)$; then $d(\alpha, \gamma)<\varepsilon$ by $(\mathrm{S}, 3)$ and $\alpha|\Delta=\gamma| \Delta$ by $(\mathrm{S}, 1)$. Now (for $r=1,2, \cdots, s-1 ; s=1,2, \cdots)$,

$$
d\left(\gamma^{s}(Q), f\left(G_{r}\right)\right)>\delta_{r}-\sum_{j=r+1}^{s} \varepsilon_{j} \geqq \delta_{r}\left(1-\sum_{j=r+1}^{s} 2^{-s+r-1}\right) \geqq \delta_{r} / 2 .
$$


Thus $\gamma(Q) \cap f\left(G_{r}\right)=\varnothing(r=1,2, \cdots)$, so that $\gamma(Q) \cap f\left(R_{k}\right)=\varnothing$.

The proof of (1.1). That $i_{*}$ is one-to-one follows from (3.2) with $Q=S^{m} \times[0,1]$ and $\Delta=\left(S^{m} \times\{0,1\}\right) \cup\left(\left\{x_{0}\right\} \times[0,1]\right)(m+k \leqq p-2)$. It is well known that, given $\alpha: S^{m} \rightarrow N^{p}, \alpha\left(x_{0}\right)=y_{0}$, there exists $\varepsilon>0$ such that, if $\gamma: S^{m} \rightarrow N^{p}, \gamma\left(x_{0}\right)=y_{0}$, then $\gamma$ is homotopic to $\alpha$ in $N^{p}$. That $i_{*}$ is onto follows from (3.2) for $Q=S^{m}$ and $\Delta=\left\{x_{0}\right\}(m+k \leqq p-1)$.

3.3. Remarks. Actually, we have proved more than (1.1) states. Given any $\varepsilon>0$ and a map or homotopy $\alpha$ in $N^{p}$, the map or homotopy $\gamma$ found in $N^{p}-f\left(R_{k}\right)$ has $d(\alpha, \gamma)<\varepsilon$.

For all $n$ and $p$ and $f: M^{n} \rightarrow N^{p}$ only $C^{n-1}(n \geqq 2),(1.1)$ is not true. Let $g_{j}: E^{j} \rightarrow E^{1}$ be the maps given by [9] with critical arcs $A_{j}$; let $H_{j}=g_{j}^{-1}((0,1))$ $\left([0,1]=g_{j}\left(A_{j}\right)\right)$, let $r:(0,1) \rightarrow E^{1}$ be a $C^{\infty}$ homeomorphism, and let $h_{j}: H_{j} \rightarrow E^{1}$ be the restriction map $r g_{j} \mid H_{j}(j=1,2, \cdots, n)$. For $p=1, h_{n}$ is the desired example $f$ since $E^{1}=h_{n}\left(R_{0}\left(h_{n}\right)\right)$. Given $p$ and $j(j=2,3, \cdots, \min (n, p) ; p=2,3, \cdots)$, define

$$
f: H_{j} \times E^{n-j} \rightarrow E^{p}
$$

by $f_{i}\left(x_{1}, x_{2}, \cdots, x_{n}\right)=h_{j}\left(x_{1}, x_{2}, \cdots, x_{j}\right)(i=1,2, \cdots, j) ; f_{i}\left(x_{1}, x_{2}, \cdots, x_{n}\right)=x_{i}$ $(i=j+1, j+2, \cdots, \min (n, p)) ; \quad$ and $f_{i}\left(x_{1}, x_{2}, \cdots, x_{n}\right)=0 \quad(i=\min (n, p)+1$, $\min (n, p)+2, \cdots, p)$. Then $f\left(R_{\min (n, p)-j}(f)\right)$ is a tame open $(\min (n, p)-j+1)$-cell in $E^{p}$, and

$$
\pi_{p-\min (n, p)+j-2}\left(E^{p}-f\left(R_{\min (n, p)-j}(f)\right)\right) \neq 0 .
$$

Also, it is easy to see that (1.1) does not hold for $f\left(R_{k}(f)\right)$ replaced by $\mathrm{Cl}\left[f\left(R_{k}(f)\right)\right]$.

4. The proof of (1.2). The set $E_{f}$ is the minimal set $E$ satisfying [3, (2.1)], and $E_{f} \subset B_{f}$ (definition in [4, p. 528]).

4.1. Lemma. Suppose that in (3.1) $n=p \geqq 3, h$ is $C^{n}$ light open, $q=n-2$, and $\operatorname{dim} Q \leqq 2($ rather than $q+\operatorname{dim} Q \leqq p-1)$. If $R_{q}(h)$ is replaced by $E_{h}$, then the corresponding conclusion holds.

Proof. We will describe only the changes in the proof of (3.1) which are required here. Of course the first paragraph of that proof is irrelevant in this case. In the second paragraph, and thereafter, $R_{q}(h)$ is replaced by $E_{h}$ (and the statement relating $R_{0}$ and $R_{q}$ is omitted).

The restriction map $h \mid A_{w}$ is light open [10, p. 147, (7.2)], and the union of the branch sets $B\left(h \mid A_{w}\right)$ is $B_{h}$ [3, proof of (2.1)]. Thus

(1) $h\left(B_{h} \cap Y\right)$, and in particular $h\left(E_{h} \cap Y\right)$, meets each plane $\{w\} \times E^{2}$ in a finite set $[10$, p. $198,(5.1)]$.

(2) Let $\Lambda=\pi\left(h\left(E_{h} \cap Y\right)\right)$; then $\Lambda$ is a compact nowhere dense subset of $E^{n-2}$ [3, proof of (2.1)]. Thus $\operatorname{dim} \Lambda \leqq n-3$ [5, p. 44]. (Actually in [3, proof of (2.1)] the analogous-and equivalent-statement for the projection of $E_{h}$ itself was shown.) 
Because of (1), the sets $G_{i}, H_{i}$, and $K_{i}$ may be defined as before, with $L=\Lambda$. Let $\beta^{1}$ be the map given by $(2.1)$ for $\alpha, v=\delta \cdot 2^{-q}, \quad V^{\prime}=E^{n}, P=P_{1}$, $E=\left(G_{1} \cap \Lambda\right) \times S_{1}(\operatorname{dim} E \leqq n-3$ by $(2))$, and $W=H_{1} \times \operatorname{int}\left(K_{1}\right)$. Thus $\beta^{1}(Q) \cap P_{1}$ is a finite set in $\operatorname{int}\left(P_{1}\right)$, and

$$
\beta^{1}(Q) \cap\left(\left(G_{1} \cap \Lambda\right) \times S_{1}\right)=\varnothing .
$$

Given any map $\beta^{i}: Q \rightarrow N^{p}$ such that $\beta^{i}(Q) \cap P_{i}$ is a finite set $\left\{a_{i, j}\right\}$ $\left(j=1,2, \cdots, k_{i}\right)$ and

$$
\beta^{i}(Q) \cap\left(\left(G_{i} \cap \Lambda\right) \times S_{i}\right)=\varnothing
$$

we now define a map $\gamma^{i}: Q \rightarrow N^{p}$. Define $\mu_{i}$ so that, in addition to its previous properties,

$$
\mu_{i}<d\left(\pi\left(a_{i, j}\right), \Lambda \cup \operatorname{bdy}\left(G_{i}\right)\right)
$$

$\left(j=1,2, \cdots, k_{i}\right)$. Define $g^{i}$ as before except on $\{w\} \times K_{i}$ for $\left(w, \pi\left(a_{i, j}\right)\right) \leqq \mu_{i}$. For each such $w$ replace each (coordinate) 2-rectangle of $K_{i}$, say of side $t$, with a coordinate 2-rectangle of the same center and side $d\left(w, \pi\left(a_{i, j}\right)\right)\left(\mu_{1}\right)^{-1} t$. Define $g^{i}$ on $\{w\} \times\left(E^{2}-S_{i}\right)$ analogously to its definition for $w$ elsewhere in $G_{i}$.

Beginning with the properties (a), (b), (c) and (d) of the map $\gamma^{i}$ obtained from $\beta^{i}$, the sets $G_{j}$ should be replaced by $G_{j} \cap \Lambda$ for the rest of the proof ( $P_{j}$ remains $\left.G_{j} \times S_{j}\right)$. The only other change is that in the definition of $\beta^{I+1}(2.1)$ is applied for $\alpha=\gamma^{I}, P=P_{I+1}, E=\left(G_{I+1} \cap \Lambda\right) \times S_{I}$,

$$
W=\left(H_{I+1} \times \operatorname{int}\left(K_{I+1}\right)\right)-\bigcup_{i=1}^{I}\left(\left(G_{i} \cap \Lambda\right) \times K_{i}\right),
$$

and $v<\delta \cdot 2^{-q}$. Then $\beta^{I+1}(Q) \cap P_{I+1}$ is a finite set, and $\beta^{I+1}(Q) \cap E=\varnothing$.

4.2. Lemma. Let $f: M^{n} \rightarrow N^{n}$ be a $C^{n}$ light open map, $n \geqq 3$, and let $Q$ be a finite polyhedron with $\operatorname{dim} Q \leqq 2$. Let $\varepsilon$ be positive, let $\Delta \subset Q$ be compact, and let $\alpha: Q \rightarrow N^{n}$ be a map such that $\alpha(\Delta) \cap f\left(E_{f}\right)=\varnothing$. Then there exists a map $\gamma: Q \rightarrow N^{n}$ such that $\gamma(Q) \cap f\left(E_{f}\right)=\varnothing, \gamma|\Delta=\alpha| \Delta$, and $d(\alpha, \gamma)<\varepsilon$.

The proof is a slight modification of that of (3.2), and we only give the changes and additions. The compact sets $A_{i}$ are defined so that $E_{f}=\bigcup_{i} A_{i}$.

Define the sets $X_{i, q, j}(q=0,1, \cdots, n-3)$ as before. Given the locally compact space $A_{i}-R_{n-3}$, the sets $A_{i} \cap W_{i, n-2, j}$ form an open cover since $E_{f} \subset B_{f} \subset R_{n-2}$ [3, (1.6)]; let $X_{i, n-2, j}$ be the closed cover of $A_{i}-R_{n-3}$ given by (2.4). As in (3.2) $G_{s}$ is compact $(s=1,2, \cdots)$.

The only other change occurs in the case that $C_{S+1}$ is a set $X_{i, n-2, j}$ (rather than $\left.X_{i, q, j} ; q=0,1, \cdots, n-3\right)$ : let $\gamma^{s+1}$ be the map given by (4.1), rather than by (3.1).

The proof of (1.2). We need only prove the last sentence [3, (2.1)]. For $n=2$ 
the conclusion is immediate since $E_{f}=\varnothing$. For $n \geqq 3$ the proof is identical to that of (1.1), except that (4.2) is used instead of (3.2) and that $m=1$.

The last statement (the extension) of (1.1) answers a question S. S. Cairns asked the author. It might be useful because locally such a map is a pseudo-covering map [4, p. 529, Definition 5 and (1.4)].

4.3. RemarK. Let $n=3$, let $V$ be a coordinate 3-cell in $N^{3}$, and let $Y$ be a compact set in $M^{3}$ such that $f(Y) \subset V$. Then the (at most) 0 -dimensional set $f\left(Y \cap E_{f}\right)$ is tame in $V$, i.e., there exists a homeomorphism $h: V \rightarrow E^{3}$ such that $h\left(f\left(Y \cap E_{f}\right)\right)$ is contained in a straight line.

By arguments analogous to those preceding one may show that, for each $\varepsilon>0$, there exist mutually disjoint polyhedral 3-cells $C_{i}(i=1,2, \cdots, m)$ such that $f\left(E_{f} \cap Y\right) \subset \bigcup \operatorname{int}\left(C_{i}\right)$. The conclusion follows from [1, pp. 435-436]. That (1.2) does not imply the conclusion follows from [6]. (The author is indebted to L. V. Keldys for supplying this reference.)

\section{REFERENCES}

1. R. H. Bing, Tame Cantor sets in E3, Pacific J. Math. 11 (1961), 435-446.

2. P. T. Church, Differentiable open maps, Bull. Amer. Math. Soc. 68 (1962), 468-469.

3. - Differentiable open maps on manifolds, Trans. Amer. Math. Soc. 109 (1963), $87-100$

4. P. T. Church and E. Hemmingsen, Light open maps on n-manifolds, Duke Math. J. 27 (1960), 527-536. 1941.

5. W. Hurewicz and H. Wallman, Dimension theory, Princeton Univ. Press, Princeton, N. J.,

6. A. Kirkor, Wild 0-dimensional sets and the fundamental group, Fund. Math. 45 (1958), 228-236.

7. J. Milnor, Differential topslogy, mimeographed notes, Princeton Univ., Princeton, N. J., 1958.

8. A. P. Morse, The behavior of a function on its critical set of points, Ann. of Math. (2) 40 (1939), 62-70.

9. H. Whitney, A function not constant on a connected set of critical points, Duke Math. J. 1 (1935), 514-517.

10. G. T. Whyburn, Analytic topology, Amer. Math. Soc. Colloq. Publ. Vol. 28, Amer. Math. Soc., Providence, R. I., 1942.

INSTITUTE FOR DefENSE ANALYSES, Princeton, New Jersey 